Pacific Journal of Mathematics

SUFFICIENCY, RMS CONDITION AND RELATIVE ENTROPY
IN YON NEWMAN ALGEBRAS 


\title{
SUFFICIENCY, KMS CONDITION AND RELATIVE ENTROPY IN VON NEUMANN ALGEBRAS
}

\author{
Fumio Hial, Masanori OHya and Makoto Tsukada
}

\begin{abstract}
The sufficiency in von Neumann algebras is discussed with some applications to classification of normal states. It is shown that the concept of sufficiency characterizes the KMS-states and the invariant states with respect to a modular automorphism group. The relations between the sufficiency and the relative entropy are established.
\end{abstract}

Introduction. Since the investigation of sufficient statistics in abstract measure theoretic terms was initiated by Halmos and Savage [10], the concept of sufficiency has been developed by many mathematical statisticians in terms of various relations given by comparison of experiments, risk functions within the framework of statistical decision problems and so on. A characterization of sufficiency was given in [12] through the measure of Kullback-Leibler information.

The concept of sufficiency was first generalized by Umegaki [22, 23 ] to the noncommutative case of semi-finite von Neumann algebras with some extension of the Kullback-Leibler information (usually called the relative entropy). Later the related discussions especially concerning the relative entropy for quantum systems have been made by several authors, e.g., Araki [2,3], Gudder and Marchand [7], and Lindblad [13].

As defined precisely and explained in $\S \S 1$ and 4 of this paper, the concept of sufficiency is more or less considered through the informativity of a certain subalgebra with respect to a given algebra for a dynamical system of interest. Namely, in the case that such a subalgebra is sufficient, the relative entropy on the subalgebra is equal to that on the given algebra. This fact may or may not be a reason why the concept of sufficiency has not been entered into analysis of physical systems, in which the change of entropy is thought of more relevant.

The Kubo-Martin-Schwinger (KMS) condition was introduced by these three authors $[11,14]$ as a boundary condition of the thermal Green function. Haag, Hugenholtz and Winnink [8] showed that in the operator algebraic framework this condition is a fundamental one describing thermal equilibrium of quantum systems. The KMS condition through the Tomita-Takesaki theory now becomes a core of studying von Neumann algebras.

Under the above historical basis, our main motivation of this 
work is as follows: How useful for quantum systems is the concept of sufficiency? How much of the related topics of sufficiency, mostly done for the commutative case, can be generalized to the noncommutative case?

Having these questions in our mind, we discuss the sufficiency with some applications to classification of normal states on the basis of recent development of von Neumann algebras.

In $\S 1$ of this paper, we establish definitions and notations used throughout and also give some simple facts.

In $\S 2$, it is shown that the concept of sufficiency characterizes the invariant states and the KMS-states with respect to a modular automorphism group.

In $\S 3$, we prove some formulas on the relative entropy using Araki's definition of relative entropy.

In $\S 4$, combining several theorems obtained in the previous sections, we establish some results which indicate the relations between the sufficiency and the relative entropy.

As a whole, we like to claim that the concept of sufficiency might be very useful for analysing von Neumann algebras and hence some quantum systems.

1. Definition and preliminaries. Throughout this paper, let $\Re$ be a von Neumann algebra with unity $I$ acting on a Hilbert space $\mathscr{H}$, and $\mathbb{S}$ be the set of all normal states of $\mathfrak{N}$. A dynamical system of physically interest is described by a triple $(\Re, \mathbb{B}, \alpha)$, where $\alpha_{t}, t \in \boldsymbol{R}$, is a strongly continuous one-parameter automorphism group of $\Re$. A state $\varphi \in \mathbb{S}$ is said to satisfy the Kubo-Martin-Schwinger (KMS) boundary condition at a certain constant $\beta>0$ with respect to $\alpha_{t}$ if for every pair $A, B \in \Re$ there exists a bounded function $F_{A, B}(z)$ continuous on and holomorphic in the strip $0 \leqq \operatorname{Im} z \leqq \beta$ with boundary values:

$$
F_{A, B}(t)=\varphi\left(\alpha_{t}(A) B\right) \text { and } F_{A, B}(t+i \beta)=\varphi\left(B \alpha_{t}(A)\right) .
$$

If $\varphi$ satisfies the KMS condition with respect to $\alpha_{t}$, then $\varphi$ is proved to be $\alpha_{t}$-invariant, i.e., $\varphi \circ \alpha_{t}=\varphi$. Considering $\alpha_{\beta t}$, we may take $\beta=1$ in the sequel discussions. Takesaki showed in [17] using Tomita's theory that to every faithful state $\phi \in \mathbb{B}$ there exists a unique one-parameter automorphism group (i.e., the so-called modular automorphism group) $\sigma_{t}^{\circ}$ with respect to which $\phi$ satisfies the KMS condition at $\beta=1$.

In this paper, a subalgebra $\mathfrak{M}$ always means a von Neumann subalgebra of $\mathfrak{N}$ with $I$. For a subalgebra $\mathfrak{M}$ and a state $\varphi \in \mathbb{B}$, let $E_{\varphi}(\cdot \mid \mathfrak{M})$ denote the conditional expectation with respect to $\mathfrak{M}$ and $\phi$ (if it exists), which is characterized as a norm one normal projec- 
tion from $\mathfrak{N}$ onto $\mathfrak{M}$ satisfying $\varphi(A)=\varphi\left(E_{\varphi}(A \mid \mathfrak{M})\right.$ ) for all $A \in \mathfrak{N}$ (cf. $[19,21])$. It was shown by Takesaki [18] that for a faithful state $\varphi \in \mathbb{B}$ the conditional expectation $E_{\varphi}(\cdot \mid \mathfrak{M})$ exists if and only if $\mathfrak{M}$ is invariant under the modular automorphism group $\sigma_{t}^{\varphi}$.

According to [5], for any two faithful states $\varphi, \psi \in(\$)$ there exists a strongly continuous function $t \mapsto u_{t}$ of $\boldsymbol{R}$ into the unitary group of $\Re$ which is a $\varphi$-cocycle, i.e.,

$$
u_{s+t}=u_{s} \sigma_{s}^{\varphi}\left(u_{t}\right), \quad s, t \in \boldsymbol{R},
$$

and which satisfies

$$
\sigma_{t}^{\psi}(A)=u_{t} \sigma_{t}^{\varphi}(A) u_{t}^{*}, \quad t \in \boldsymbol{R}, \quad A \in \Re .
$$

This $\varphi$-cocycle $u_{t}$ is denoted by $u_{t}=(D \psi: D \varphi)_{t}$ and is called the Connes Radon-Nikodym derivative of $\psi$ with respect to $\varphi$. Some discussions are found in [4, 9] concerning Connes Radon-Nikodym derivatives and conditional expectations.

Let $S$ be a subset of $\mathbb{S}$. A subalgebra $\mathfrak{M}$ is said to be sufficient for $S$ if $E_{\varphi}(\cdot \mid \mathfrak{M})$ exists for each $\varphi \in S$ and for every $A \in \mathfrak{R}$ there exists an $A_{0} \in \mathfrak{M}$ such that

$$
\left.A_{0}=E_{\varphi}(A \mid \mathfrak{M}) \text { a.e. [ } \varphi\right], \quad \varphi \in S,
$$

where $A=B$ a.e. $[\varphi]$ means $\varphi(|A-B|)=0$. This definition of sufficient subalgebras is somewhat weaker than that in [22]. Also we call $\mathfrak{M}$ to be minimal sufficient for $S$ if $\mathfrak{M}$ is sufficient for $S$ and any subalgebra being sufficient for $S$ includes $\mathfrak{M}$.

For $\varphi, \psi \in \mathcal{B}$, it is said that $\psi$ is absolutely continuous with respect to $\varphi$ (we write $\psi \ll \varphi$ ) if for each $A \in \mathfrak{R}, \varphi\left(A^{*} A\right)=0$ implies $\psi\left(A^{*} A\right)=0$; that is, $\psi \ll \varphi$ if and only if $s(\psi) \leqq s(\varphi)$ where $s(\varphi)$ is the support projection of $\varphi$. We give here the elementary facts of sufficiency which are readily seen from the definition.

$\left(1^{\circ}\right)$ Let $\varphi, \psi \in \mathbb{B}$ with $\psi \ll \varphi$. Then a subalgebra $\mathfrak{M}$ is sufficient for $\{\varphi, \psi\}$ if and only if $E_{\varphi}(\cdot \mid \mathfrak{M})$ exists and $\psi(A)=\psi\left(E_{\varphi}(A \mid \mathfrak{M})\right)$ for all $A \in \Re$.

$\left(2^{\circ}\right)$ If a subalgebra $\mathfrak{M}$ is sufficient for $\{\varphi, \psi\}$, then $\varphi=\psi$ on $\mathfrak{R}$ if and only if $\varphi=\psi$ on $\mathfrak{M}$.

When $S(\subset \mathbb{B})$ contains a faithful state $\varphi$, then:

$\left(3^{\circ}\right)$ A subalgebra $\mathfrak{M}$ is sufficient for $S$ if and only if $\mathfrak{M}$ is sufficient for every pair $\{\phi, \psi\}$ with $\psi \in S$.

$\left(4^{\circ}\right)$ If $\mathfrak{M}$ is sufficient for $S$, then any subalgebra $\mathfrak{M}_{1}$ including $\mathfrak{M}$ is sufficient for $S$ whenever $E_{\varphi}\left(\cdot \mid \mathfrak{M}_{1}\right)$ exists.

2. Sufficiency and characterization of states. The following lemma is a restatement of [4, Lemma 1.6] in our terminology. We 
give the proof for completeness.

LEMma 2.1. For each subalgebra $\mathfrak{M}$ and two faithful states $\varphi, \psi \in \mathbb{S}$, the following conditions are equivalent:

(i) $\mathfrak{M}$ is sufficient for $\{\varphi, \psi\}$;

(ii) $E_{\varphi}(\cdot \mid \mathfrak{M})$ exists and $(D \psi: D \varphi)_{t} \in \mathfrak{M}$ for every $t \in \boldsymbol{R}$.

Proof. Let $\hat{\varphi}=\varphi \uparrow \mathfrak{M}$ and $\hat{\psi}=\psi \uparrow \mathfrak{M}$. Assume that $\mathfrak{M}$ is sufficient for $\{\varphi, \psi\}$. Then the conditional expectation $E_{\varphi}(\cdot \mid \mathfrak{M})$ exists and $\psi(A)=\psi\left(E_{\varphi}(A \mid \mathfrak{M})\right)$ for $A \in \mathfrak{R}$. By [5, Lemma 1.4.4], we have

$$
(D \psi: D \varphi)_{t}=(D(\hat{\psi} \circ E): D(\hat{\varphi} \circ E))_{t}=(D \hat{\psi}: D \hat{\varphi})_{t} \in \mathfrak{M}
$$

for every $t \in R$, where $E(\cdot)=E_{\varphi}(\cdot \mid \mathfrak{M})$. Conversely assume that $E_{\varphi}(\cdot \mid \mathfrak{M})$ exists and $(D \psi: D \varphi)_{t} \in \mathfrak{M}$ for all $t \in \boldsymbol{R}$. Since $\boldsymbol{\sigma}_{t}^{\hat{\varphi}}=\sigma_{t}^{\varphi} \uparrow \mathfrak{M}$, it follows that $u_{t}=(D \psi: D \varphi)_{t}$ is a $\hat{\varphi}$-cocycle. By [5, Theorem 1.2.4], there exists a unique faithful normal semi-finite weight $\widetilde{\psi}$ on $\mathfrak{M}$ such that $(D \tilde{\psi}: D \hat{\varphi})_{t}=u_{t}$. Define a faithful normal semi-finite weight $\psi^{\prime}$ on $\mathfrak{N}$ by $\psi^{\prime}(A)=\widetilde{\psi}\left(E_{\varphi}(A \mid \mathfrak{M})\right)$ for $A \in \mathfrak{\Re}$. Then it follows that

$$
\left(D \psi^{\prime}: D \varphi\right)_{t}=(D \tilde{\psi}: D \hat{\varphi})_{t}=(D \psi: D \varphi)_{t}, \quad t \in \boldsymbol{R} .
$$

Hence we have $\psi^{\prime}=\psi$, so that $\psi(A)=\psi\left(E_{\varphi}(A \mid \mathfrak{M})\right)$ for every $A \in \mathfrak{R}$. This shows that $\mathfrak{M}$ is sufficient for $\{\varphi, \psi\}$.

In this section, let $\varphi$ be a fixed faithful normal state of $\Re$ and $\sigma_{t}^{\circ}$ its modular automorphism group. Let $Z_{\varphi}$ be the subalgebra consisting of all $A \in \mathfrak{N}$. such that $\varphi(A B)=\varphi(B A)$ for every $B \in \mathfrak{N}$. The subalgebra $Z_{\varphi}$ is called the centralizer of $\varphi$ and is exactly the fixed point algebra of $\sigma_{t}^{\varphi}$ (cf. [17, Lemma 15.8]), i.e.,

$$
Z_{\varphi}=\left\{A \in \Re: \sigma_{t}^{\varphi}(A)=A, t \in R\right\} .
$$

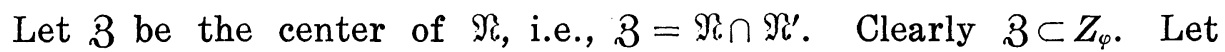
$I(\varphi)$ be the set of all $\sigma_{t}^{\varphi}$-invariant states in $\mathcal{B}$, and $K(\varphi)$ be the set of all states in $(S)$ satisfying the KMS condition with respect to $\sigma_{t}^{\varphi}$ at $\beta=1$. Then we have:

TheOREM 2.2. (1) For each $\psi \in \mathbb{S}, \psi \in I(\varphi)$ if and only if $Z_{\varphi}$ is sufficient for $\{\varnothing, \psi\}$.

(2) The centralizer $Z_{\varphi}$ is minimal sufficient for $I(\varphi)$.

Proof. (1) Let $\psi \in \mathbb{B}$ and take $\psi_{1}=(\psi+\varphi) / 2$. Then we easily see that $\psi \in I(\varphi)$ is equivalent to $\psi_{1} \in I(\varphi)$, and the sufficiency of $Z_{\varphi}$ for $\{\varphi, \psi\}$ is equivalent to that for $\left\{\varphi, \psi_{1}\right\}$. Therefore we can assume that $\psi$ is faithful. Since $Z_{\varphi}$ is elementwise invariant under $\sigma_{t}^{\varphi}$, 
there exists the conditional expectation $E_{\varphi}\left(\cdot \mid Z_{\varphi}\right)$ from $\mathfrak{N}$ onto $Z_{\varphi}$. Hence, in view of Lemma 2.1, it suffices to show that $\psi \in I(\varphi)$ if and only if $(D \psi: D \varphi)_{t} \in Z_{\varphi}$ for every $t \in \boldsymbol{R}$. If $\psi \in I(\varphi)$, then by [5, Lemma 1.2.3] there exists a positive self-adjoint operator $h$ affiliated with $Z_{\varphi}$ such that $(D \psi: D \varphi)_{t}=h^{i t} \in Z_{\varphi}$ for all $t \in \boldsymbol{R}$. Conversely suppose that $(D \psi: D \varphi)_{t} \in Z_{\varphi}$ for every $t \in \boldsymbol{R}$. Since

$$
\sigma_{t}^{\psi}(A)=(D \psi: D \varphi)_{t} \sigma_{t}^{\varphi}(A)(D \psi: D \varphi)_{t}^{*},
$$

we have

$$
\varphi\left(\sigma_{t}^{\psi}(A)\right)=\varphi\left(\sigma_{t}^{\varphi}(A)\right)=\varphi(A), \quad A \in \mathfrak{R} .
$$

Hence it follows that $\varphi$ is $\sigma_{t}^{\psi}$-invariant, and thus $\psi$ is $\sigma_{t}^{\varphi}$-invariant (cf. [17, Theorem 15.2]).

(2) It follows from (1) that $Z_{\varphi}$ is sufficient for every pair $\{\varphi, \psi\}$ with $\psi \in I(\varphi)$. Hence $Z_{\varphi}$ is sufficient for $I(\varphi)$. To show the minimality of $Z_{\varphi}$, let $\mathfrak{M}$ be any subalgebra which is sufficient for $I(\varphi)$. We now prove that $Z_{\varphi} \subset \mathfrak{M}$. Take any positive invertible operator $h \in Z_{\varphi}$ with $\varphi(h)=1$, and define a faithful state $\psi \in \mathbb{B}$ by $\psi(A)=\varphi(h A)$ for $A \in \Re$. Then we have $\psi \in I(\varphi)$ and $(D \psi: D \varphi)_{t}=h^{i t}$. Since $(D \psi: D \varphi)_{t} \in \mathbb{M}$ for every $t \in \boldsymbol{R}$ by Lemma 2.1, it follows that $h \in \mathfrak{M}$. Thus $Z_{\varphi} \subset \mathfrak{M}$.

TheOREM 2.3. (1) For each $\psi \in \mathbb{B}, \psi \in K(\varphi)$ if and only if 3 is sufficient for $\{\varphi, \psi\}$.

(2) The center 3 is minimal sufficient for $K(\varphi)$.

Proof. As in the proof of Theorem 2.2, we can assume that $\psi$ is faithful. If $\psi \in K(\varphi)$, then by [15, Theorem 5.4] there exists a positive self-adjoint operator $h$ affiliated with 3 such that $\psi(A)=$ $\varphi(h A)$ for $A \in \Re$, so that $(D \psi: D \varphi)_{t}=h^{i t} \in 3$ for every $t \in \boldsymbol{R}$. Conversely if $(D \psi: D \varphi)_{t} \in \mathcal{Z}$ for every $t \in \boldsymbol{R}$, then by (2.1) we have $\sigma_{t}^{\psi}=\sigma_{t}^{\varphi}$ and hence $\psi \in K(\varphi)$. Thus (1) is proved. The proof of (2) is analogous to that of Theorem 2.2.

3. Relative entropy. When $\mathfrak{N}$ is finite dimensional, for each $\varphi$ and $\psi$ in $\&$ the relative entropy $S(\varphi \mid \psi)$ is defined by

$$
S(\varphi \mid \psi)=\operatorname{tr}\left(\rho_{\psi} \log \rho_{\psi}-\rho_{\psi} \log \rho_{\varphi}\right),
$$

where $\rho_{\varphi}$ and $\rho_{\psi}$ are density matrices for $\varphi$ and $\psi$. Araki [2,3] extended the relative entropy to the case for normal positive linear functionals of general von Neumann algebras, and studied its several properties such as joint convexity, lower semiconitinuity and monotonicity. 
In this section, we assume as in [3] that $\mathfrak{N}$ has a cyclic and separating vector. Let $V$ be a natural positive cone (cf. [1]) for $\mathfrak{R}$ and let $\varphi$ and $\psi$ be states in (\$). By [1, Theorem 6], there exist unique vector representatives $\Phi$ and $\Psi$ of $\varphi$ and $\psi$ in $V$ such that $\varphi(A)=\langle\Phi, A \Phi\rangle$ and $\psi(A)=\langle\Psi, A \Psi\rangle$ for all $A \in \mathfrak{R}$. The operator $S_{\Phi, \Psi}$ with the domain

$$
D\left(S_{\Phi, \Psi}\right)=\mathfrak{N} \Psi+\left(I-s^{\Re^{\prime}}(\Psi)\right) \mathscr{H}
$$

is defined by

$$
S_{\phi, \Psi}(A \Psi+\Omega)=s^{\Re}(\Psi) A^{*} \Phi, \quad A \in \Re, \quad s^{\Re^{\prime}}(\Psi) \Omega=0,
$$

where $s^{\Re}(\Psi)(\in \mathfrak{R})$ denotes the $\mathfrak{R}$-support of the vector $\Psi$. Then $S_{\Phi, \Psi}$ is a closable conjugate-linear operator (cf. [3]) and the relative modular operator $\Delta \Phi, \psi$ is defined by

$$
\Delta \varphi, \Psi=\left(S_{\phi, \Psi}\right)^{*} \overline{S_{\phi, \Psi}} .
$$

The relative entropy $S(\varphi \mid \psi)$ is now given by

$$
S(\varphi \mid \psi)= \begin{cases}-\langle\Psi,(\log \Delta \varphi, \Psi) \Psi\rangle & \text { if } \psi \ll \varphi, \\ +\infty & \text { otherwise }\end{cases}
$$

Then $S(\varphi \mid \psi) \geqq 0$, and $S(\varphi \mid \psi)=0$ if and only if $\varphi=\psi$. For each subalgebra $\mathfrak{M}$, let $S_{\mathfrak{N}}(\varphi \mid \psi)$ denote the relative entropy of the restrictions of $\varphi$ and $\psi$ to $\mathfrak{M}$. By the monotonicity of relative entropy generally proved in [20], it holds that

$$
S_{\sqrt{2}}(\varphi \mid \psi) \leqq S(\varphi \mid \psi)
$$

for every subalgebra $\mathfrak{M}$ (also see $[2,3,13,23]$ ).

THEOREM 3.1. For each $\varphi, \psi \in \mathbb{B}$,

$$
\|\varphi-\psi\| \leqq\{2 S(\varphi \mid \psi)\}^{1 / 2}
$$

Proof. By [16, p.31], we can take two normal positive linear functionals $\varphi_{1}$ and $\varphi_{2}$ such that $\varphi-\psi=\varphi_{1}-\varphi_{2},\|\varphi-\psi\|=\left\|\varphi_{1}\right\|+$ $\left\|\varphi_{2}\right\|$ and $s\left(\varphi_{1}\right) \perp s\left(\varphi_{2}\right)$. Let $e=s\left(\varphi_{1}\right)$. Then it follows that

$$
\begin{aligned}
\|\varphi-\psi\| & =(\varphi-\psi)(e)-(\varphi-\psi)(I-e) \\
& =2(\varphi(e)-\psi(e)) .
\end{aligned}
$$

Let $\mathfrak{M}$ be the subalgebra generated by $e$ and $I-e$. By using the monotonicity, we have

$$
\begin{aligned}
S(\varphi \mid \psi) & \geqq S_{\mathfrak{m}}(\varphi \mid \psi) \\
& =\psi(e) \log \frac{\psi(e)}{\varphi(e)}+\psi(I-e) \log \frac{\psi(I-e)}{\varphi(I-e)} .
\end{aligned}
$$


It was shown in [6] that

$$
2|\alpha-\beta| \leqq\left\{2\left(\beta \log \frac{\beta}{\alpha}+(1-\beta) \log \frac{1-\beta}{1-\alpha}\right)\right\}^{1 / 2}
$$

for $0 \leqq \alpha, \beta \leqq 1$. Taking $\alpha=\varphi(e)$ and $\beta=\psi(e)$, we deduce the desired inequality.

TheOREM 3.2. Let $\varphi, \psi \in(B)$ be faithful and $\mathfrak{M}$ be a subalgebra such that $\mathfrak{M} \subset Z_{\varphi}$. Define $\psi^{\prime} \in \mathbb{S}$ by $\psi^{\prime}(A)=\psi\left(E_{\varphi}(A \mid \mathfrak{M})\right)$ for $A \in \mathfrak{N}$. If $S_{\mathfrak{M}}(\varphi \mid \psi)<+\infty$, then

$$
S\left(\psi^{\prime} \mid \psi\right)=S(\varphi \mid \psi)-S_{\mathfrak{\Downarrow i}}(\varphi \mid \psi)
$$

Proof. First note that $\psi^{\prime}$ is well defined from $\mathfrak{M} \subset Z_{\varphi}$. Since $\varphi \uparrow \mathfrak{M}$ is a faithful normal trace, there exists a positive self-adjoint operator $h$ affiliated with $\mathfrak{M}$ such that $\psi(A)=\varphi(h A)$ for all $A \in \mathfrak{M}$. Take the spectral decomposition $h=\int_{0}^{\infty} \lambda \operatorname{de}(\lambda)$ and $h_{n}=\int_{0}^{n} \lambda \operatorname{de}(\lambda)$. Since $h_{n} \in \mathfrak{M}$, we have for every $A \in \mathfrak{N}$

$$
\begin{aligned}
\psi^{\prime}(A) & =\psi\left(E_{\varphi}(A \mid \mathfrak{M})\right)=\lim _{n \rightarrow \infty} \varphi\left(h_{n} E_{\varphi}(A \mid \mathfrak{M})\right) \\
& =\lim _{n \rightarrow \infty} \varphi\left(E_{\varphi}\left(h_{n} A \mid \mathfrak{M}\right)\right)=\lim _{n \rightarrow \infty} \varphi\left(h_{n} A\right)=\varphi(h A) .
\end{aligned}
$$

Hence it follows (cf. [5, Lemma 1.2.3]) that $\left(D \psi^{\prime}: D \varphi\right)_{t}=h^{i t}$ for all $t \in \boldsymbol{R}$. By the relations

$$
\begin{aligned}
\left(D \psi^{\prime}: D \psi\right)_{t}=\left(D \psi^{\prime}: D \varphi\right)_{t}(D \varphi: D \psi)_{t}=h^{i t}(D \varphi: D \psi)_{t}, \\
\\
\left(D \psi^{\prime}: D \psi\right)_{t}=\left(\Delta_{\psi^{\prime}, \psi}\right)^{i t}{\bar{\psi}^{-i t}}^{-i}, \\
(D \varphi ; D \psi)_{t}=\left(\Delta_{\mathscr{q}, \Psi}\right)^{i t} \Delta_{\Psi}^{-i t},
\end{aligned}
$$

where $\Delta \Psi=\Delta \Psi, \Psi$, we deduce that $\left(\Delta_{\Psi}, \Psi\right)^{i t}=h^{i t}\left(\Delta_{\phi, \Psi}\right)^{i t}$ for all $t \in \boldsymbol{R}$. Moreover since $h^{i t} \in \mathfrak{M} \subset Z_{\varphi}$ and

$$
\sigma_{t}^{\varphi}(A)=\left(\Delta_{\Phi, \psi}\right)^{i t} A\left(\Delta_{\Phi, \Psi}\right)^{-i t}, \quad A \in \Re,
$$

it follows that $h^{i t}$ and $\left(\Delta_{\Phi, \Psi}\right)^{i t}$ commute. Now let $\hat{\Phi}$ and $\hat{\Psi}$ be vector representatives of $\varphi \uparrow \mathfrak{M}$ and $\psi \uparrow \mathfrak{M}$ in a natural positive cone $\hat{V}$ for $\mathfrak{M}$. Since $\varphi \backslash \mathfrak{M}$ is a trace, it follows that $\Delta_{\hat{\psi}, \hat{\varphi}}=h$. By $\log \Delta_{\hat{\phi}, \hat{\psi}}=-$ $J\left(\log \Delta_{\hat{\psi}}, \hat{p}\right) J$ where $J$ is the modular conjugation operator associated with $\hat{V}$ (cf. [3, Remark 3.4]), we have

$$
\begin{aligned}
S_{\mathfrak{m}}(\varphi \mid \psi) & =-\left\langle\hat{\Psi},\left(\log \Delta_{\hat{\varphi}, \hat{\Psi}}\right) \hat{\Psi}\right\rangle \\
& =\langle\hat{\Psi},(\log h) \hat{\Psi}\rangle=\langle\Psi,(\log h) \Psi\rangle,
\end{aligned}
$$

which is finite from the assumption. Therefore we obtain

$$
S\left(\psi^{\prime} \mid \psi\right)=-\left\langle\Psi,\left(\log \Delta_{\Psi^{\prime}, \Psi}\right) \Psi\right\rangle
$$




$$
\begin{aligned}
& =-\left\langle\Psi,\left(\log \Delta_{\varphi, \Psi}\right) \Psi\right\rangle-\langle\Psi,(\log h) \Psi\rangle \\
& =S(\varphi \mid \psi)-S_{\mathfrak{m}}(\varphi \mid \psi) .
\end{aligned}
$$

THEOREM 3.3. Let $\varphi \in(B)$ be faithful and $\mathfrak{M}$ be a subalgebra such that $\mathfrak{M} \subset Z_{\varphi}$. Let $\psi \in \mathbb{S}$ and define $\psi^{\prime} \in \mathbb{S}$ by $\psi^{\prime}(A)=\psi\left(E_{\varphi}(A \mid \mathfrak{M})\right)$ for $A \in \mathfrak{N}$. Suppose either (a) $\psi$ is faithful or (b) $\psi \leqq \lambda \varphi$ for some $\lambda>0$. If $S_{\mathfrak{m}}(\varphi \mid \psi)<+\infty$, then

$$
\left\|\psi^{\prime}-\psi\right\| \leqq\left\{2\left(S(\varphi \mid \psi)-S_{\mathfrak{m}}(\varphi \mid \psi)\right)\right\}^{1 / 2}
$$

Proof. For the case (a), the desired inequality is immediate from Theorems 3.1 and 3.2. Now suppose that $\psi \leqq \lambda \varphi$ for some $\lambda>0$. For each $\varepsilon>0$, let $\psi_{\varepsilon}=(1+\varepsilon)^{-1}(\psi+\varepsilon \phi) \in(S)$ and define $\psi_{\varepsilon}^{\prime} \in(S)$ by $\psi_{\varepsilon}^{\prime}(A)=\psi_{\varepsilon}\left(E_{\varphi}(A \mid \mathfrak{M})\right)$. By the convexity of relative entropy (cf. [3, Theorem 3.8]), we have

$$
S_{\mathfrak{m}}\left(\varphi \mid \psi_{\varepsilon}\right) \leqq(1+\varepsilon)^{-1} S_{\mathfrak{m}}(\varphi \mid \psi)<+\infty .
$$

Hence it follows from the case (a) that

$$
\left\|\psi_{\varepsilon}^{\prime}-\psi_{\varepsilon}\right\| \leqq\left\{2\left(S\left(\varphi \mid \psi_{\varepsilon}\right)-S_{\mathfrak{M}}\left(\varphi \mid \psi_{\varepsilon}\right)\right)\right\}^{1 / 2} .
$$

Since $\psi_{\varepsilon} \leqq \lambda \varphi$ for each $\varepsilon>0$, by [3, Theorem 3.7] we have

$$
\begin{aligned}
& \lim _{\varepsilon \rightarrow+0} S\left(\varphi \mid \psi_{\varepsilon}\right)=S(\varphi \mid \psi), \\
& \lim _{\varepsilon \rightarrow+0} S_{\mathfrak{M}}\left(\varphi \mid \psi_{\varepsilon}\right)=S_{\mathfrak{R}}(\varphi \mid \psi) .
\end{aligned}
$$

Since $\psi_{\varepsilon}^{\prime}=(1+\varepsilon)^{-1}\left(\psi^{\prime}+\varepsilon \varphi\right)$, we obtain the desired inequality by letting $\varepsilon \rightarrow+0$ in (3.3).

Before closing this section, we have to note that Professor Araki gave us very important comments to some results of our first version of this paper, which make us enable to write them in the above form.

4. Sufficiency and relative entropy. In this section, let a faithful state $\varphi \in \mathbb{S}$ be fixed as in $\S 2$.

THEOREM 4.1. For each subalgebra $\mathfrak{M} \subset Z_{\varphi}$ and each $\psi \in \mathbb{B}$, the following statements hold:

(1) Suppose the condition (a) or (b) in Theorem 3.3. If $\mathfrak{M}$ is sufficient for $\{\varphi, \psi\}$, then $S_{\mathfrak{m}}(\phi \mid \psi)=S(\varphi \mid \psi)$, and conversely if $S_{\mathfrak{M}}(\varphi \mid \psi)=S(\varphi \mid \psi)<+\infty$, then $\mathfrak{M}$ is sufficient for $\{\varphi, \psi\}$.

(2) If $\mathfrak{M}$ is sufficient for $\{\varphi, \psi\}$, then $S_{\mathfrak{m}}(\varphi \mid(\psi+\varphi) / 2)=$ $S(\varphi \mid(\psi+\varphi) / 2)$, and conversely if $S_{\mathfrak{m}}(\varphi \mid(\psi+\varphi) / 2)=S(\varphi \mid(\psi+\varphi) / 2)<+\infty$, then $\mathfrak{M}$ is sufficient for $\{\varphi, \psi\}$. 
Proof. (1) We use the notations in the proof of Theorem 3.2. Let $\mathfrak{M}$ be sufficient for $\{\varphi, \psi\}$, and suppose the condition (a). There exists a positive self-adjoint operator $h$ affiliated with $\mathfrak{M}$ such that $\psi(A)=\varphi(h A)$ for all $A \in \mathfrak{M}$. Then we have

$$
\psi(A)=\psi\left(E_{\varphi}(A \mid \mathfrak{M})\right)=\varphi(h A), \quad A \in \mathfrak{R},
$$

as in the proof of Theorem 3.2. Hence it follows that $(D \psi: D \varphi)_{t}=$ $h^{i t}$, and we have

$$
\left(\Delta_{\varphi, \Psi}\right)^{i t}=(D \varphi: D \psi)_{t} \Delta_{\Psi}^{i t}=h^{-i t} \Delta_{\Psi}^{i t} .
$$

Since $h^{i t}$ and $\left(\Delta_{\Phi, \Psi}\right)^{i t}$ commute, it follows that $h^{-i t}$ and $\Delta_{\Psi}^{i t}$ commute. We thus have

$$
\begin{aligned}
S(\varphi \mid \psi) & =-\left\langle\Psi,\left(-\log h+\log \Delta_{\Psi}\right) \Psi\right\rangle \\
& =\langle\Psi,(\log h) \Psi\rangle,
\end{aligned}
$$

by $\Delta_{\Psi} \Psi=\Psi$. From (3.2) and (4.1), we obtain $S_{\mathfrak{n}}(\varphi \mid \psi)=S(\varphi \mid \psi)$. The case (b) is proved from the case (a) by taking limits as in the proof of Theorem 3.3.

Assume conversely that $S_{\mathfrak{n}}(\varphi \mid \psi)=S(\varphi \mid \psi)<+\infty$. Then it follows from Theorem 3.3 that $\psi^{\prime}=\psi$, which implies that $\mathfrak{M}$ is sufficient for $\{\phi, \psi\}$.

(2) is immediate from (1), since $\mathfrak{M}$ is sufficient for $\{\varphi, \psi\}$ if and only if $\mathfrak{M}$ is sufficient for $\{\varphi,(\psi+\varphi) / 2\}$.

The above fact (1) extends the result [23, Theorem 5] which was proved under some strong assumptions. Combining Theorem 4.1 with Theorems 2.2 and 2.3 , we have the following:

CoRollary 4.2. (1) Suppose the condition (a) or (b) in Theorem 3.3. If $\psi \in I(\varphi)$, then $S_{Z_{\varphi}}(\varphi \mid \psi)=S(\varphi \mid \psi)$, and conversely if $S_{Z_{\varphi}}(\varphi \mid \psi)=S(\varphi \mid \psi)<+\infty$, then $\psi \in I(\varphi)$.

(2) If $\psi \in I(\varphi)$, then $S_{Z_{\varphi}}(\varphi \mid(\psi+\varphi) / 2)=S(\varphi \mid(\psi+\varphi) / 2)$, and conversely if $S_{Z \varphi}(\varphi \mid(\psi+\varphi) / 2)=S(\varphi \mid(\psi+\varphi) / 2)<+\infty$, then $\psi \in I(\varphi)$.

COROLLARY 4.3. (1) Suppose the condition (a) or (b) in Theorem 3.3. If $\psi \in K(\varphi)$, then $S_{3}(\varphi \mid \psi)=S(\varphi \mid \psi)$, and conversely if $S_{3}(\varphi \mid \psi)=$ $S(\varphi \mid \psi)<+\infty$, then $\psi \in K(\varphi)$.

(2) If $\psi \in K(\varphi)$, then $S_{8}(\varphi \mid(\psi+\varphi) / 2)=S(\varphi \mid(\psi+\varphi) / 2)$, and conversely if $S_{3}(\varphi \mid(\psi+\varphi) / 2)=S(\varphi \mid(\psi+\varphi) / 2)<+\infty$, then $\psi \in K(\varphi)$.

The monotonicity (3.1) says that the restriction of measurement to a subalgebra $\mathfrak{M}$ usually makes it more difficult to discriminate between two states. From Theorem 4.1, the physical meaning of suf- 
ficiency might be explained as follows: If a subalgebra $\mathfrak{M}$ is sufficient for $\{\varphi, \psi\}$, then we obtain from the measurement of $\mathfrak{M}$ as much information as from that of $\mathfrak{N}$ to discriminate between $\varphi$ and $\psi$. In particular, to distinguish $\psi \in I(\varphi)$ (resp. $\psi \in K(\varphi)$ ) from $\varphi$, the measurement of $Z_{\varphi}$ (resp. 3) gives as much information as $\mathfrak{R}$.

ACKNOWLEDGMENT. The authors wish to express their gratitude to Professor H. Umegaki for his valuable advice and constant encouragement. They thank Professor $\mathrm{H}$. Araki for his critical reading and essential comments for this work. Professor J. Tomiyama and the referee kindly indicated the useful paper [4] to the authors.

\section{REFERENCES}

1. H. Araki, Some properties of modular conjugation operator of von Neumann algebras and a non-commutative Radon-Nikodym theorem with a chain rule, Pacific J. Math., 50 (1974), 309-354.

2. - Relative entropy of states of von Neumann algebras, Publ. RIMS, Kyoto Univ., 11 (1976), 809-833.

3. - Relative entropy for states of von Neumann algebras II, Publ. RIMS, Kyoto Univ., 13 (1977), 173-192.

4. F. Combes and C. Delaroche, Groupe modulaire d'une espérance conditionnelle dans une algébre de von Neumann, Bull. Soc. Math. France, 103 (1975), 385-426.

5. A. Connes, Une classification des facteurs de type III, Ann. Sci. Ecole Norm. Sup. Sér. 4, 6 (1973), 133-252.

6. I. Csiszár, Information-type measures of difference of probability distributions and indirect observations, Studia Sci. Math. Hungar., 2 (1967), 299-318.

7. S. Gudder and J.-P. Marchand, Noncommutative probability on von Neumann algebras, J. Math. Phys., 13 (1972), 799-806.

8. R. Haag, N. M. Hugenholtz and M. Winnink, On the equilibrium states in quantum statistical systems, Commun. Math. Phys., 5 (1967), 215-236.

9. U. Haagerup, Operator valued weights in von Neumann algebras, I, J. Functional Analysis, 32 (1979), 175-206.

10. P. R. Halmos and L. J. Savage, Application of the Radon-Nikodym theorem to the theory of sufficient statistics, Ann. Math. Statistics, 20 (1949), 225-241.

11. R. Kubo, Statistical mechanical theory of irreversible processes I, J. Phys. Soc. Japan, 12 (1957), 570-586.

12. S. Kullback and R. A. Leibler, On information and sufficiency, Ann. Math. Statistics, 22 (1951), 79-86.

13. G. Lindblad, Expectations and entropy inequalities for finite quantum systems, Commun. Math. Phys., 39 (1974), 111-119.

14. P. C. Martin and J. Schwinger, Theory of many-particle systems I, Phys. Rev., 115 (1959), 1342-1373.

15. G. K. Pedersen and M. Takesaki, The Radon-Nikodym theorems for von Neumann algebras, Acta Math., 130 (1973), 53-87.

16. S. Sakai, $C^{*}$-Algebras and $W^{*}$-Algebras, Springer, Berlin, 1971.

17. M. Takesaki, Tomita's Theory of Modular Hilbert Algebras and its Applications, Springer, Lecture notes in math. Vol. 128, 1970.

18. - Conditional expectations in von Neumann algebras, J. Functional Analysis, 9 (1972), 306-321. 
19. J. Tomiyama, On the projection of norm one in $W^{*}$-algebras, Proc. Japan Acad., 33 (1957), 608-612.

20. A. Uhlmann, Relative entropy and the Wigner-Yanase-Dyson-Lieb concavity in an interpolation theory, Commun. Math. Phys., 54 (1977), 21-32.

21. H. Umegaki, Conditional expectation in an operator algebra, Tôhoku Math. J., 6 (1954), 177-181.

22. - Conditional expectation in an operator algebra, III, Ködai Math. Sem. Rep., 11 (1959), 51-64.

23. - Conditional expectation in an operator algebra, IV (entropy and information), Kōdai Math. Sem. Rep., 14 (1962), 59-85.

Received March 13, 1979 and in revised form January 4, 1980.

SCIENCE UNIVERSITY OF TOKYO

Noda City, Chiba 278, JapaN 



\section{PACIFIC JOURNAL OF MATHEMATICS}

\section{EDITORS}

DONALD BABBITT (Managing Editor)

University of California

Los Angeles, California 90024

Hugo RossI

University of Utah

Salt Lake City, UT 84112

C. C. MOORE and ANDREW OGG

University of California

Berkeley, CA 94720

\section{J. DugundJI}

Department of Mathematics University of Southern California Los Angeles, California 90007

R. FinN and J. Milgram Stanford University Stanford, California 94305

\section{ASSOCIATE EDITORS}
R. ARENS
E. F. BECKENBACH
B. H. Neumann
F. WOLF
K. YoshidA

\section{SUPPORTING INSTITUTIONS}

UNIVERSITY OF ARIZONA

UNIVERSITY OF BRITISH COLUMBIA

CALIFORNIA INSTITUTE OF TECHNOLOGY

UNIVERSITY OF CALIFORNIA

MONTANA STATE UNIVERSITY

UNIVERSITY OF NEVADA, RENO

NEW MEXICO STATE UNIVERSITY

OREGON STATE UNIVERSITY
UNIVERSITY OF OREGON

UNIVERSITY OF SOUTHERN CALIFORNIA

STANFORD UNIVERSITY

UNIVERSITY OF HAWAII

UNIVERSITY OF TOKYO

UNIVERSITY OF UTAH

WASHINGTON STATE UNIVERSITY

UNIVERSITY OF WASHINGTON 


\section{Pacific Journal of Mathematics}

\section{Vol. 96, No. $1 \quad$ November, 1981}

Hédi Amara, Groupe des classes et unité fondamentale des extensions quadratiques relatives à un corps quadratique imaginaire principal $\ldots \ldots \ldots 1$

Douglas S. Bridges, On the isolation of zeroes of an analytic function $\ldots \ldots 13$ Andrew J. Casson and John L. Harer, Some homology lens spaces which

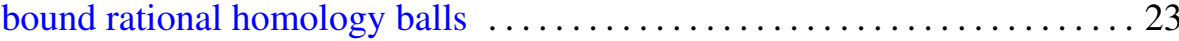

Z. A. Chanturia, On the absolute convergence of Fourier series of the classes $H^{\omega} \cap V[v]$

J.-F. Colombeau and Mário Carvalho Matos, On some spaces of entire functions defined on infinite-dimensional spaces $\ldots \ldots \ldots \ldots \ldots \ldots \ldots 63$

Edwin Duda, Pointwise periodic homeomorphisms on chainable continua . . .77

Richard F. Gustafson, A simple genus one knot with incompressible spanning surfaces of arbitrarily high genus $\ldots \ldots \ldots \ldots \ldots \ldots \ldots \ldots 1$

Fumio Hiai, Masanori Ohya and Makoto Tsukada, Sufficiency, KMS condition and relative entropy in von Neumann algebras

Ted Hurley, Intersections of terms of polycentral series of free groups and free Lie algebras. II .................................. 111

Robert Edward Jamison, II, Partition numbers for trees and ordered sets . . 115 R. D. Ketkar and N. Vanaja, A note on FR-perfect modules ............. 141 Michihiko Kikkawa, On Killing-Ricci forms of Lie triple algebras ....... 153

Jorge Lewowicz, Invariant manifolds for regular points 163

Richard W. Marsh, William H. Mills, Robert L. Ward, Howard Rumsey and Lloyd Richard Welch, Round trinomials .....

Claude Schochet, Topological methods for $C^{*}$-algebras. I. Spectral sequences

Yong Sian So, Polynomial near-fields?

Douglas Wayne Townsend, Imaginary values of meromorphic functions in the disk

Kiyoshi Watanabe, Coverings of a projective algebraic manifold .. 243

Martin Michael Zuckerman, Choosing $l$-element subsets of $n$-element sets 